\title{
An Experimental and Simulation Studies of a High Strain-Rate Deformation Shear Band in a High-Nickel Steel
}

\author{
Sung-Il Baik, ${ }^{1,2}$ Dieter Isheim, ${ }^{1,2}$ Divya Jain, ${ }^{1}$ R. K. Gupta, ${ }^{3}$ K.S. Kumar, ${ }^{3}$ David N Seidman ${ }^{1,2,{ }^{*}}$ \\ 1. Department of Materials Science and Engineering, Northwestern University, Evanston, IL 60208, USA \\ 2. Northwestern University Center for Atom Probe Tomography (NUCAPT), 2220 Campus Drive, \\ Evanston, IL 60208, USA \\ ${ }^{3}$ School of Engineering, Brown University, Providence, RI 02912, USA
}

A high Ni-steel was developed for both high-strength and -toughness for cryogenic temperature applications [1]. High-Ni steels are used for severe working conditions, such as blast-resistant naval ship hulls or decks and liquefied natural-gas (LNG) tanks. Even though this particular high-Ni steel possesses excellent static properties, a thermoplastic instability in the form of localized shear bands is a major concern for high-speed deformation conditions. The adiabatic shear-band (ASB) is a specific type of deformation in a narrow zone that occurs at a high strain-rate with a significant localized temperature increase [2]. There isn't sufficient time for thermal diffusion from a local volume, resulting in a significant temperature increase in a localized zone. This results in the suppression of work hardening and localized softening occurs, which can cause material failures. Atomic-scale observations indicate grain segmentation, growth and solute redistribution at ASBs, which are caused by a severe deformation and local temperature increase on a short time-scale [3]. The interaction between solute atoms with dislocations and grain boundaries (GBs) is the source of the instability of mechanical properties. The temperature increase causes segregation of solute atoms at dislocations and GBs, and the supersaturation of solute may be sufficiently high for precipitates to nucleate.

The current research explores the ferrite-austenite phase transformation and atomic-scale redistribution of solute atoms at an ASB. The ASB is analyzed by correlative experiments: electron backscatter diffraction (EBSD), transmission electron microscopy (TEM), atom-probe tomography (APT), and the thermodynamic and kinetic calculations. The material studied has a nominal composition of $0.32 \mathrm{C}-0.04 \mathrm{~N}-0.61 \mathrm{Mn}-0.41 \mathrm{Si}-0.13 \mathrm{Cu}-0.64 \mathrm{Cr}-0.64 \mathrm{Mo}-0.08 \mathrm{~V}-0.005 \mathrm{Nb}-9.75 \mathrm{Ni}-$ Bal. Fe (at.\%). The dynamic compression deformation tests with strain rates of $2800 \mathrm{~s}^{-1}$, employing a split-Hopkinson confirmed that this alloy forms ASBs.

Fig.1 displays the results of correlative experiments employing SEM, TEM, EBSD, and 3-D APT to study a specific region of interest of a shear band, failure area or crack tip in the high-Ni steel. The width of the shear band in front of the crack tip is about $10 \mu \mathrm{m}$ as observed by SEM, Fig.1(a). The crack has propagated along the ASB. The local temperature increase, with a hot-spot region developing in the ASB, facilitates nucleation of voids and ductile fracture occurs within the ASB. Fig.1(b,c) display a plan-view bright-field (BF) TEM image and a high-resolution EBSD map in the ASB region after using the dual-beam FIB microscope lift-out preparation technique to target the rectangular region indicated in Fig.1(a) for analysis. These TEM and EBSD analyses illustrate the change of grain shape across the ASB: from elongated lamellar grains to equiaxed grains with high misorientation angle as the center of the shear band is approached. This transition from elongated lamellar grains to equiaxed small grains is indicated by a white or blue dotted line in Fig. 1(b) or (c), respectively. The breakdown of deformed elongated grains into smaller grains or subgrains is an important process in ASB formation. Fig. 1(d,e) is a correlative analysis by TEM and 3-D APT of equiaxed small grains in the core of an ASB. TEM and 
3-D APT analyses indicate the redistribution of solute atoms during the ASB deformation. The analyses intersect the dark grains in the TEM image, which are enriched in carbon (black dots in (e)) and other austenite stabilizing elements. Vanadium-rich precipitates are also observed in the ferrite matrix in the core of ASB. The size and the density of V-rich nitride precipitate are $5-7 \mathrm{~nm}$ diameter and $2.2 \times 10^{23} \mathrm{~m}^{-3}$ respectively. The thermodynamic and kinetic behavior of $\mathrm{VN}(\mathrm{C})$ precipitation was studied by austenite/carbonitride equilibrium model [4]. The precipitation kinetic is accelerated by up to two orders of magnitude faster in a severely deformed conditions during dynamic compression deformation.

\section{References}

[1] G.R. Brophy, A.J. Miller, T Am Soc Metal, 41 (1949) 1185-1203.

[2] P. Wang, K.S. Kumar, Mat Sci Eng a-Struct, 519 (2009) 184-197.

[3] R.W. Armstrong, S.M. Walley, Int Mater Rev, 53 (2008) 105-128.

[4] P. Maugis, M. Goune, Acta materialia, 53 (2005) 3359-3367.

[5] This research is funded by the Office of Naval Research under grants N00014-12-1-0425, N000141110681, Dr. W. Mullins, grant manager. The atom-probe tomography measurements were performed at the Northwestern University Center for Atom-Probe Tomography (NUCAPT). The LEAP 4000X-Si tomograph was purchased and upgraded with funding from NSF-MRI (DMR-0420532) and ONR-DURIP (N00014-0400798, N00014- 0610539, N00014-0910781).

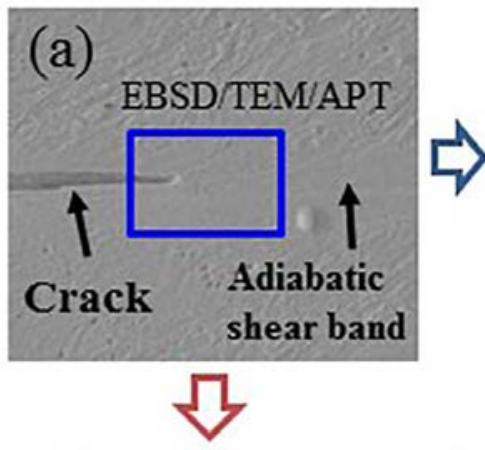

Atom probe tomography

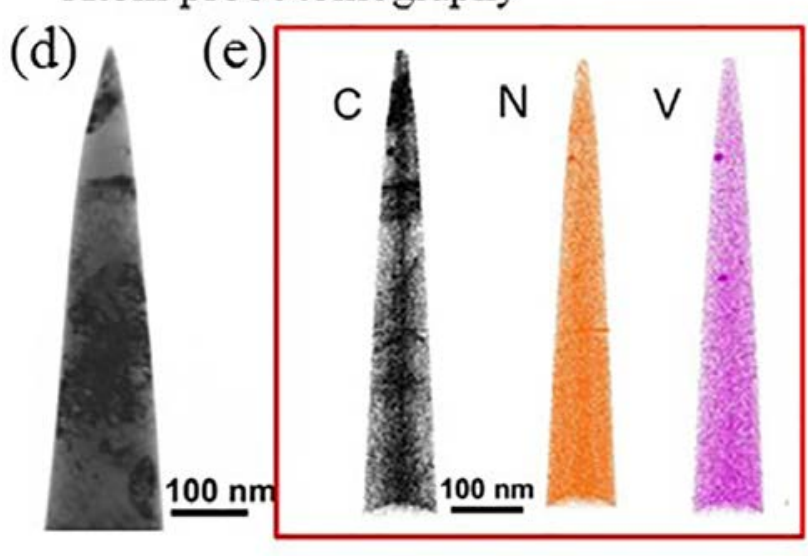

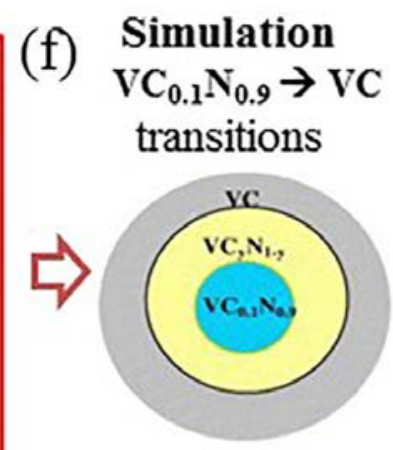

Kinetics of vanadium carbonitride precipitation in steel: A computer model [4]

Fig. 1: The transition of the grain structure from elongated lath grains on a mesoscale and the formation of austenite phase and carbonitride on an atomic scale are found in this correlative study using (a) SEM, (b, d) TEM, (c) EBSD, and (e) APT analyses. Equiaxed small grains and VN-type precipitates are formed during the temperature increase in the course of the ASB formation during high strain-rate deformation. The precipitate behavior is also understood using thermodynamic and kinetic calculation for a steel [4]. 